\title{
Glocalisation, runaway et local production
}

Deux ou trois choses que je sais d'elles

Glocalization, Runaway and local Production. Two or three things I know about them

Joël Augros

\section{OpenEdition}

\section{Journals}

Édition électronique

URL : http://journals.openedition.org/questionsdecommunication/1774

DOI : 10.4000/questionsdecommunication. 1774

ISSN : 2259-8901

\section{Éditeur}

Presses universitaires de Lorraine

\section{Édition imprimée}

Date de publication : 1 juillet 2008

Pagination : 225-238

ISBN : $978-2-86480-952-4$

ISSN : 1633-5961

Référence électronique

Joël Augros, «Glocalisation, runaway et local production », Questions de communication [En ligne], 13। 2008, mis en ligne le 01 juillet 2010, consulté le 08 avril 2021. URL : http://journals.openedition.org/ questionsdecommunication/1774; DOI : https://doi.org/10.4000/questionsdecommunication.1774 


\section{$>$ ÉCHANGES}

JOËL AUGROS

Groupe de recherche sur l'économie du cinéma et de l'audiovisuel

Université Paris 8 Vincennes-Saint-Denis

joel.augros@univ-paris8.fr

\section{GLOCALISATION, RUNAWAYET LOCAL PRODUCTION. DEUX OU TROIS CHOSES QUE JE SAIS D'ELLES}

Résumé. - En matière de cinéma, coexistent plusieurs formes de relation entre marché local et marché international. Cet article focalise le regard sur deux de ces relations, autour du concept de glocalisation : la runaway production et la local production. Sont examinés les éléments historiques qui caractérisent ces formes de production cinématographique et leur situation actuelle. En écho aux articles de Joëlle Farchy et Jean Tardif (Questions de communication, I3, 2008), sont abordés ensuite les notions d'avantages comparatifs, de régulation et de captation de marchés de niche à l'aune de ces runaway et local productions.

Mots clés. - Glocalisation, Local production, runaway production, Hollywood, avantages comparatifs, régulation. 
e sujet n'occupe pas toujours la une des gazettes, pourtant nombre de feuillets ont été noircis pour considérer, discuter, apprécier les implications de la mondialisation ou globalisation de la culture. L'ouvrage de Joëlle Farchy et Jean Tardif publié en 2006, Les enjeux de la mondialisation culturelle, avait l'immense mérite de préciser les données de ce débat, d'en donner les cadres conceptuels mais aussi de tracer des perspectives d'action. Les textes de ces deux auteurs publiés dans cette livraison de Questions de communication ont toutes les qualités de cette somme. Aussi le propos de l'article ne sera-t-il pas de discuter ces textes pas à pas, mais d'alimenter et d'illustrer le débat à partir d'un angle précis, sur un marché délimité. Cette contribution s'intéresse aux articulations entre le marché international et les marchés locaux en matière de cinéma. À partir de cet axe, nous discuterons librement de certains points développés par Jean Tardif et Joëlle Farchy.

\section{Des diverses formes de glocalisation en matière de cinéma}

Le mot glocalisation est un mot valise qui marie globalisation et localisation selon le mantra «think globally and act locally 》. Le terme serait né à la fin des années 80 au Japon et utilisé rapidement dans le cadre du marketing. Son acception traduit alors le fait d'adapter un produit global aux réalités locales. Mais le concept est ensuite repris par certains sociologues comme Roland Robertson et transcrit l'idée d'une vision plus complexe que celle de la globalisation, permettant d'y insérer les aspects sociaux et culturels. En matière de cinéma, la notion n'est pas l'apanage des major companies hollywoodiennes, mais fut, par exemple. explicitement avancée par les dirigeants de Canal Plus à la fin des années 90 , lors d'une tentative d'expansion européenne où la compagnie française tentait de marier les activités de plusieurs sociétés par la voie d'accords ou de prises de participations (Pathé en Grande-Bretagne et en France, Tobis en Allemagne, Sogepaq en Espagne, accords de coproduction en Italie, Working Title en Grande-Bretagne). Les professionnels étatsuniens utilisent peu le terme de glocalisation, sans doute parce que, de fait, les majors hollywoodiennes font depuis les années 20 de la glocalisation comme Monsieur Jourdain faisait de la prose (adaptation des campagnes marketing dans les différents pays, choix parmi l'ensemble de la production des films qui conviennent au marché local, etc.).

Outre la question de la distribution des films étrangers sur un marché donné - qui ne nous intéresse pas ici -, en synthèse, les liens entre marché global et marché locaux ressortissent de trois stratégies 
différentes: runaway production, local production (production localisée)' et coproduction. Nous examinerons compendieusement les deux premières, sans omettre de préciser qu'il existe entre celles-ci des zones de contact et d'interaction. La runaway production est, en substance, une forme de décentralisation ou délocalisation de la production à l'étranger. Une situation guère neuve, nous y reviendrons. Cependant, la plus grande partie de l'histoire hollywoodienne s'est fondée sur la présence en Californie, particulièrement en Californie du Sud, de l'essentiel des moyens de production du cinéma; les films arborant fièrement après le The End final la mention Made in Hollywood, USA. À cela une raison : alors que la production - télévision et film - requiert des technologies de plus en plus complexes, le regroupement d'une masse critique de professionnels qualifiés et de sociétés de service liées au secteur du divertissement dans la région de Los Angeles a généré des externalités qui ont fait de la Californie du Sud une zone attractive pour les productions à bas coût: «En raison de cette combinaison d'économies d'échelle et d'externalité géographique, la Californie du Sud importe des acteurs, réalisateurs et professionnels qualifiés du monde entier tout en exportant des films et des programmes de TV dans le reste du monde » (Chase, $2000: 4$ ). Malgré la concentration des moyens de financement, des meilleurs artistes, des compagnies de production, de post-production, de péri-production dans la région de Los Angeles, le phénomène de runaway production s'est mis en place dès les premiers temps du cinéma, de façon concomitante mais limitée. En effet, celle-ci est alors essentiellement engendrée par la recherche de paysages ou de main d'œuvre à bon marché, notamment pour les films épiques qui requièrent un nombre important de figurants (Espagne, Italie où Rome devient Hollywood-sur-Tibre²).

Les tournages de films opérés dans ce cadre à partir des années 90 découlent de phénomènes nouveaux. Alors même que les atouts anciens de la délocalisation des tournages peuvent maintenant être simulés par ordinateurs - c'est le cas des armées de figurants, c'est aussi celui des paysages (López, 2004) -, les nouveauxavantages proviennent de l'édiction d'aides financières (Canada, Australie), de la création ou la rénovation de studios de tournage performants (Espagne, Maroc, Tchéquie), mais aussi de l'installation d'un tissu de techniciens suffisamment qualifiés (GrandeBretagne, Tchéquie). De plus, l'évolution des télécommunications permet de continuer à travailler avec des compagnies de post-production sises à

\footnotetext{
' Pour distinguer de «production locale» qui dans l'acception courante signifie la production de films autochtones.

${ }^{2}$ Le cas du film de M. Scorsese, Gangs of New York (2002), est assez symptomatique, le réalisateur affirmant dans la presse que le tournage à Cinecittà lui permet de faire le dernier film épique avec de vrais figurants.
} 
Los Angeles sans éprouver la nécessité d'une présence sur place (la vision des dailies, le développement des effets spéciaux de post-production, la mise en place de la musique, entre autres opérations, peuvent s'effectuer par transferts informatiques rapides et peu onéreux).

On assiste donc à une amplification de la délocalisation des tournages qui désertent Los Angeles pour s'installer au Canada (Hollywood North), mais aussi en Australie, au Maroc, à Malte, en Allemagne... 33 \% des films conçus aux États-Unis seraient tournés en dehors du pays selon un rapport de 2005 de la Los Angeles Economic Development Corporation (Wright, 2006)3 ${ }^{3}$ Les productions sont attirées hors des États-Unis par des incitations fiscales alléchantes. Un film tourné à Ciudad de la Luz, en Espagne peut, sous certaines conditions, obtenir une aide de 7 millions de dollars. En Allemagne, le tournage de Walkyrie au studio de Babelsberg a été favorisé par une contribution de 7,14 millions de dollars, dans un pays où les aides peuvent, là encore selon les circonstances, atteindre 20 \% du budget initial $\left.\right|^{4}$. Une concurrence sévère s'instaure frisant le dumping fiscal. À la mise en place des aides canadiennes répond des avantages fiscaux en Australie qui entraînent, à leur tour, l'ouverture de schémas fiscaux encore plus favorables par l'État fédéral ou les provinces canadiennes. En retour, les aides des États ou de villes aux États-Unis même (aides qui ne sont pas inexistantes) sont augmentées : «Ce qui rend ces politiques attractives pour les gouvernements étrangers - et menaçantes pour Hollywood - est que la production de divertissement (entertainment), à la différence de beaucoup de services, n'est pas attaché à un lieu spécifique. Les inputs dans le processus de production sont libres comme l'air en ce que les ressources peuvent être transportées d'un lieu à un autre pour le tournage » (Chase, 2000 : 20). L'essentiel du capital (acteurs, réalisateurs, stars) est mobile et le marché des lieux de tournage très volatil. Ainsi les producteurs de The Chronicle of Narnia: the Lion, the Witch and the Wardrobe (2005) ont-ils songé à tourner successivement en Tchéquie, en Grande-Bretagne, en Irlande, aux Canada, aux États-Unis avant d'élire un studio néo-zélandais. II serait cependant malvenu de considérer la runaway production comme un phénomène singulier du cinéma hollywoodien. Des équipes française se retrouvent périodiquement à Prague au grand dam des industries techniques hexagonales; des équipes indiennes tournent en Nouvelle-Zélande, à l'lle Maurice, à Singapour, ou bien encore en Suisse pour bénéficier de paysages somptueux ou de conditions de tournages avantageuses.

3 Des données que Cl. Wright (2006) indique elle-même comme étant sujet à caution puisque pour la plupart compilées et fournies par des instances opposées à ce phénomène.

${ }^{4}$ Variety (05/02/07) et reuters.com (04/02/08). 
A priori, on peut penser que cette évolution n'a que peu d'effets sur le cinéma local, sur la culture locale. En effet, les films sont destinés à être réexportés et Popeye (1980) de Robert Altman n'a rien pris de la culture maltaise ni ne lui a rien apporté, hormis un vague attraction pour les touristes désœuvrés. De plus, remarque Claire Wright (2006), les films faits dans ce cadre ne doivent pas se démarquer de films faits aux États-Unis. Si tel n'était pas le cas, selon toute vraisemblance, les producteurs étatsuniens cesseraient de tourner au Canada. Cependant, cette politique a quelques effets d'entraînement. En premier lieu, la capacité du personnel autochtone est améliorée. Cela touche aussi bien les techniciens (éclairagistes costumiers, directeurs de la photo, etc.) que les cadres (producteurs exécutifs, directeurs de production) et même les acteurs qui tous apprennent et profitent du savoir faire étranger en travaillant sans interruption, en « se frottant » aux nouvelles techniques de tournage ou de gestion. Éventuellement, les États qui accueillent ces tournages en profitent pour monter des écoles (Maroc).

En second lieu, l'allocation des ressources aux films internationaux peut s'accomplir au détriment des œuvres nationales (studios occupés par des tournages internationaux, emploi des meilleurs techniciens sur des films internationaux mieux rémunérés au détriment des productions locales, limitation des investissements dans les films locaux). Le cinéma américain est divisé sur la runaway production. Les syndicats et les États fédérés, notamment la Californie, demandent des actions de l'État fédéral. Les dirigeants des majors, aussi bien pour leur production cinéma que pour leur production télévisuelle, ainsi que quelques indépendants s'accommodent fort bien d'un système qui leur permet de baisser très substantiellement leurs coûts.

La local production, ou production localisée, est le second aspect du processus de glocalisation. II ne s'agit cependant pas seulement de s'adapter aux conditions locales, mais de produire dans et pour un marché donné. Les productions localisées sont donc constituées de films produits pas des compagnies étrangères dans un pays donné. Mais à la différence de la runaway production, ces films visent le marché local. Quand on tourne Star Wars dans les studios de Shepperton et Elstree, on ne prétend pas confectionner un film britannique pour le marché britannique, mais un film hollywoodien dont le monde est le marché. En revanche, The Full Monty (1997), produit par la Twentieth Century-Fox, « au goût anglais », répond à la définition. La distinction entre les deux catégories n'est pas toujours limpide, notamment sur le marché britannique. De la même façon, Un long dimanche de fiançailles (2004), financé par des capitaux 
étatsuniens est un film français 5 . Marathon Man (1976) ou Ronin (1998) tous deux tournés en France et financés par des capitaux étatsuniens sont des films hollywoodiens.

Cette différenciation peut paraître inutilement sémantique... Mais elle est importante car on part de deux axes très différents. Dans le premier cas, le choix de tourner dans tel ou tel pays n'a rien à voir avec le marché cinématographique de ce pays mais avec les facilités, les studios de production, la disponibilité d'équipes techniques, l'existence de facilités fiscales, le coût de la main d'œuvre, le temps, les paysages. Parmi ces atouts, il peut y avoir un lien avec le marché du cinéma. Comme vu précédemment, l'existence d'équipes techniques performantes est liée souvent à l'existence d'un cinéma local qui produit en quantité suffisante.

L'autre axe part, au contraire, du marché local. C'est parce qu'il existe un cinéma local, parce qu'une partie du public continue à s'intéresser à ses acteurs, à ses récits que l'on va lui proposer du cinéma local. Le plus souvent, les films ainsi produits ne sont pas destinés au marché international ou même au marché. Peu importe à ce niveau que cet intérêt pour le cinéma local soit « naturel » ou encadré, favorisé par des quotas ou des aides gouvernementales comme les dirigeants de la MPAA ont pu le dénoncer ${ }^{6}$.

La troisième voie est celle de la coproduction. Nous avons choisi à dessein de ne pas la traiter ici parce que d'autres questions seraient alors introduites qui dépasseraient le cadre de cette contribution et impliqueraient une discussion plus ample sur les différentes formes: de la coproduction artistique à la coproduction purement financière, des interventions ou non de la régulation étatique via des accords intergouvernementaux de production, de la question de la proximité des marchés (voir Miller, 2005).

\section{Histoire}

Si la runaway production est devenu un enjeu politique, notamment en Californie au cours des années 90, précédant d'assez peu l'annonce par les compagnies hollywoodiennes du lancement de leur stratégie de

\footnotetext{
${ }^{5}$ Ce film ne l'est plus selon la réglementation, mais l'est sans ambages aux yeux du public. Nous y reviendrons.

6 La Motion Picture Association of America est le syndicat regroupant les six major companies hollywoodiennes, organisées en groupe de pression.
} 
local production, le phénomène n'en est pas moins ancien, initié dès les débuts du cinématographe. En matière de cinéma, la mondialisation est en effet établie dès l'origine, favorisée par une donné intrinsèque à la production cinématographique : le coût de reproduction d'un film est infime par rapport à son coût de fabrication. Au cours du siècle, les deux phénomènes se sont entremêlés sans cesse.

Éclair, Star Film, Pathé, Gaumont ouvrent des succursales aux États-Unis dès les premières années $d u X X^{e}$ siècle. Ces implantations ont trois buts distincts : protéger les films de la piraterie (c'est notamment le cas de la Star Films de Georges Méliès), établir des comptoirs de vente des films faits en France, produire sur place des bandes destinées aux publics locaux et adaptées à leurs goûts. Un des premiers films faits par Pathé aux États-Unis en 1910 sera un western, The Girl from Arizona (Norden, 1994). À l'inverse, le premier film américain fait en France serait Absinthe de Herbert Brenon dès 1913 (Koszarski, 1990).

Depuis, les circonstances de mise en place de telle ou telle stratégie de production fluctuent au gré des facteurs historiques et des opportunités. Ainsi les productions locales sont-elles favorisées en Grande-Bretagne avant la Seconde Guerre mondiale par l'instauration de quotas. On tourne alors à Londres et dans la campagne anglaise à tour de bras des quota quickies. Après 1945, la production de films étrangers est favorisée par les taux de change, les régulations du travail et le gel de fonds en Europe, notamment en Grande-Bretagne mais aussi, dans une moindre mesure, en France. À cela s'ajoutent, dans d'autres pays, les coûts minimes des figurants et des constructions de décors. Cette vague comporte peu de films A, mais surtout des films B ou spectaculaires. Aux fabricants de Alexandre le Grand (1955) la production aurait annoncé à propos des dépenses potentielles : « Seul le ciel est la limite » (Lev, 1993 : 23).

Dans les années 60 et 70, les compagnies hollywoodiennes modifient leur politique. Et au lieu de simplement tourner des films étatsuniens en Europe, elles investissent dans des films tournés dans d'autres langues que l'anglo-américain. Mike Francovich de Columbia Pictures, déclare en 1961 au journaliste de Variety, Vincent Canby : «Si les Italiens veulent des films italiens, nous allons leur en fournir » (Lev, 1993 : 24). Une série d'accords entre compagnies hollywoodiennes et producteurs européens s'ensuit. Une production débute marquée par quelques grands films spectaculaires «à costumes», mais aussi des œuvres de qualité, dont certaines s'appuient ici et là sur de jeunes auteurs récemment révélés par les diverses nouvelles vagues. En Grande-Bretagne, on tourne dans ces circonstances Tom Jones (1963), A Hard Day's Night (1964), Blow Up (1966). En France, François Truffaut trouve le financement de Baisers volés (1968), L'Enfant sauvage (1970) et La Sirène du Mississipi (1969) auprès de 
compagnies hollywoodiennes, tout comme Louis Malle pour deux films ou Philippe De Broca (L'Homme de Rio, 1964). En Italie, Fellini (Satyricon, 1969, et Roma, 1972) ou Pasolini (Le Décaméron, 197I ; Salo, 1975 ; Les mille et une nuits, 1974) réalisent une partie de leur œuvre dans ces circonstances ${ }^{7}$. Ainsi, pour l'année 1968, sur 90 millions de lires investies dans le cinéma italien, 24 proviennent-elles des compagnies américaines (et 45 de coproducteurs européens), le solde étant apporté par des capitaux italiens (22 milliards) (Lev, 1993 : 65).

\section{Développement contemporain des productions locales}

Le principe des « productions localisées » a été quelque peu abandonné dans les années 80. Dans les années 90 et au début des années 2000, le phénomène a repris, sur une aire géographique vaste. On trouve des cas de productions localisées sous forme de coproductions, de productions intégrales ou d'avances-distributeurs dans nombre de pays. Ainsi en Espagne, Warner coproduit-elle quelques films et finance Reinas à 100 \%. La major est aussi distributrice, ainsi qu'en Italie, des films de Pedro Almodovar. En Allemagne, Columbia produit Anatomie (2000) alors que Warner s'investit dans Nur aus Liebe (1996), Die Furchtlosen Vier (1997) ou Kai Rabe gegen die Vatikankiller (1998) parmi d'autres films. Mais on retrouve aussi des occurrences au Japon, en Chine, à Hongkong, en Argentine, aux Pays Bas (accord de coproduction entre Warner et Bros), sans oublier le cas particulier de la Grande-Bretagne... En Inde, en 2007, Sony distribue Saawariya (2007), le premier film que la compagnie a elle-même produit sur place. Le relevé établi par Nolwenn Minguant pour sa thèse qu'elle prépare fait apparaître 49 cas pour Sony-Columbia entre 1995 et 2007, et un nombre identique pour Warner sur la même période, 39 pour Disney, 6 pour la Twentieth Century-Fox 8 ... Warner, en 2004, disait dépenser entre 75 et 100 millions de dollars par an pour les productions localisées (LaPorte, 2004).

En France, c'est en 2002 que Richard Fox (vice président de Warner Bros) et Lorenzo di Bonaventura (président de la production internationale de la même major) annoncent dans la presse la mise en place d'une politique de production de cinq à six films par an. Ils annoncent des projets avec

\footnotetext{
7 || semble qu'il y ait aussi l'influence d'un homme, E. Pleskow, qui représente à partir de 195I United Artists en Afrique du Sud, en Allemagne puis en France jusqu'en 1962 et est un élément moteur de l'adoption de cette stratégie (Stolz, 2004).

8 Nous reprenons ici les travaux de N. Mingant, thèse en cours de rédaction et G. Visco Comandini (2006).
} 
Claude Miller, Cédric Klapisch, Jean-Pierre Jeunet et un accord pour la distribution d'un minimum de quatre films avec Les Films Christian Fechner: «L'ouverture internationale permet à ce qui se fait le mieux à l'étranger d'avoir accès à Hollywood» déclarent-ils. Et ils ajoutent: «Quand nous avons commencé à produire en France, on a d'abord reçu des centaines de scripts anglais. Les gens n'avaient pas compris notre démarche qui est réellement de s'appuyer sur les talents français [...]. Dans notre esprit, c'est le marché local qui doit fonctionner $\gg$.

La stratégie est également mise en place dans le cadre de la production télévisée, surtout dans des pays où les major companies ont d'ores et déjà établies leurs propres chaînes de télévision. Ainsi, en 2007, Sony Pictures Television International produit-il au Mexique avec des partenaires locaux une telenovela Zorro : la espada y la rosa (O'Boyle, 2007). Sony Pictures Television a également un rôle étendu en Inde en raison notamment de sa chaîne sur place qu'elle doit bien nourrir (Frater, 2007). C'est aussi le cas, en partie, pour News Corp en Chine. Comme nous l'avons indiqué, la particularité de ces opérations est d'être tournées en priorité vers le marché local. La Classe de neige (1998) de Claude Miller ou L'ex-femme de ma vie (2004) de Josiane Balasko, films français qui existent grâce à l'implication de la filiale française de Warner, sont d'abord et avant tout destinés au marché national. II existe quelques cas où le film guigne un marché plus large, international. C'est le cas pour Un long dimanche de fiançailles (2004) en France ou pour le cas chinois de Tigre et dragon (2000) de Ang Lee, produit par la filiale de Columbia à Hongkong. Ces films peuvent alors disposer d'un budget plus important que la moyenne nationale.

Outre le fait de bénéficier des recettes sur le marché local, les productions localisées procurent d'autres avantages. Elles permettent de trouver de nouveaux talents, à l'instar de Paz Vega vedette de Di que sì (2004) film espagnol de Sony puis de Spanglish (2004), film hollywoodien de la même firme. Bénéficiant de cadeaux fiscaux et de coûts de prestations techniques moins élevés, les films sont plus facilement rentables. L'attribution de la nationalité autochtone peut permettre de contourner des quotas. Enfin, outre leur rentabilité possible, les intérêts à concocter un film dans ce cadre peuvent dépasser le seul appât du gain, mais se traduire par une insertion dans les réseaux locaux et permettre de peser d'une manière ou d'une autre sur les politiques locales.

9 Le Film Français, édition courriel Cannes ( 18/05/02). 


\section{Discussion}

Joëlle Farchy rappelle que, dès 1817, David Ricardo théorise les avantages comparatifs du commerce international. Kerry Chase (2000: 5) explicite en ce qui concerne le cinéma: «En matière de production cinématographique, le plus important facteur de production est le capital humain ; Amérique du nord, Europe occidentale, Asie du nord-est en sont dotés. Mais historiquement, c'est Hollywood qui a bénéficié des avantages comparatifs ». Ainsi, si la région de Los Angeles ne dispose pas d'avantages naturels, l'histoire a-t-elle fait d'Hollywood un pôle majeur de production grâce à un phénomène d'économie d'échelle initié par la taille du marché intérieur et l'établissement dans la ville d'une masse critique de talents.

Mais rien n'indique que cet avantage « naturel » puisse toujours persister. Trois raisons majeures peuvent être avancées. La théorie des avantages comparatifs postule que les biens fabriqués dans les pays défavorisés sont de qualité inférieure. Or, dans le cas de la production cinématographique comme pour toute activité culturelle, le produit intègre des aspects culturels qui en deviennent constitutifs. Le fondateur d'Universal, Carl Laemmle, voyant un western Pathé, compagnie alors dominatrice sur le marché mondial, relevait que le réalisateur français avait oublié que les Indiens n'arboraient pas de splendides moustaches comme les Apaches de Vincennes (Puttnam, 1997). L'avantage comparatif dont disposaient, avant la Première Guerre mondiale, les firmes françaises, ne suffisaient pas à rendre ipso facto la production de meilleure qualité, par la simple omission d'un trait culturel. D'autre part, les phénomènes de production délocalisée (runaway production) et des productions localisées (local production) introduisent du jeu dans l'équilibre des forces. La montée en puissance du cinéma commercial indien (Bollywood) enfin, dont les productions se coulent depuis quelques années dans les circuits commerciaux occidentaux, montre que le centre de gravité du cinéma mondial peut se déplacer à plus ou moins longue échéance. De façon assez symptomatique, des cinéastes du Bengale ou du Kerala se sont élevés contre la toute puissance du cinéma hindi, cinéma dominant dans le sous-continent. Un phénomène « d'impérialisme culturel » régional proche de celui que Joëlle Farchy reprenant Arjun Appadurai (1990, 1996) évoque à propos du processus d'indonésisation.

Joëlle Farchy sous-titre son article: «Les limites des formes actuelles de régulation ». Elle conte la lutte de la MPAA et du gouvernement des ÉtatsUnis pour combattre lors des négociations du GATT puis au sein de l'OMC les velléités protectionnistes d'un certain nombre de pays en matière de culture en général et de cinéma en particulier. La défaite de la MPAA et du gouvernement des États-Unis à la conclusion de l'Uruguay Round sur le front de l'audiovisuel a entraîné un changement de stratégie certain. Jack Valenti, 
longtemps négociateur en chef de la MPAA, déclare dès 1995 : « Nous voulons collaborer et coopérer avec les réalisateurs et les créateurs de chacun des États européens. Leurs lois, leurs quotas, leurs aides sont leur affaire. Nous regardons vers le futur, non le passé » (Warner, 1996: 8). Quand, deux ans plus tard, Claude Miller s'apprête à tourner La Classe de neige, grâce aux capitaux de la Warner, il s'empresse de déclarer: «Je ne passe pas dans le camp des Américains. Cet accord illustre au contraire le triomphe de l'exception culturelle, les Américains ont accepté le concept de cinéma national » (Dacbert, 1997 : 3). Quand, par la suite, Warner créé une filiale française, 2003 Productions, qui finance L'ex-femme de ma vie de Josiane Balasko et Un long dimanche de fiançailles de Jean-Pierre Jeunet, l'opinion exprimée par Claude Miller peut sembler fondée. Cependant, c'est aller un peu vite en besogne : on peut simplement voir dans le phénomène des productions localisées une forme de contournement de la législation locale et d'accès aux subsides gouvernementaux. Dès 1927, les quotas quickies britanniques déjà évoqués constituent un exemple intéressant. La production de films britanniques par des compagnies étrangères ouvre le droit à cellesci de distribuer un ou plusieurs de leurs propres films. Loin de constituer un détournement du principe des quotas, le quota quicky est en quelque sorte inscrit dans la loi elle-même. Tel n'est pas le cas en Espagne où, dans les années 80 , des quotas impliquent qu'un distributeur ne peut obtenir une licence d'importation qu'en échange de la distribution d'un film espagnol. En conséquence, les filiales espagnoles des compagnies hollywoodiennes ont alors tendance à accaparer la distribution des films espagnols les plus porteurs au détriment des compagnies locales, puis éventuellement de basculer vers la production de ces films, par le jeu d'avances-distributeurs en premier lieu. Enfin - dernier exemple ici présenté -, en 2005, Warner signe un contrat en Chine avec la société publique China Film Group et le studio privé Hengdian. L'opération permet à Warner d'opérer dans le pays comme un producteur local et d'échapper ainsi au quota officieux établi par les autorités chinoises (Frater, 2007). Le contournement de la barrière des quotas est une stratégie si évidente que quand Miramax, filiale de Disney, annonce son plan de production en Europe, Claudine Mulard (1994) écrit : « S'agissant de Disney, on peut aussi y voir une habile opération de relations publiques visant à atténuer l'image négative de Euro Disney ou la mise en œuvre d'une stratégie visant à contourner la barrière des quotas »».

Mais, le changement de stratégie que traduit le développement des productions localisées est dû également à deux autres phénomènes. D'abord, un changement de priorité de l'action de la MPAA. Celle-ci juge dorénavant que la lutte contre la piraterie audiovisuelle doit constituer sa principale préoccupation. Or, cette lutte nécessite la coopération des États étrangers, particulièrement pour la mise en place de forces de police spécialisées ou l'adoption de législations plus restrictives. Nous ne nous y attarderons pas. Le second phénomène est le maintien de résistances locales que mettent en relief 
et Joëlle Farchy et Jean Tardif. À cet égard, la stratégie peut être lue comme une prise en compte des niches: «Les taux de rentabilité exigés dans les grands groupes les conduisent structurellement à abandonner les créneaux les plus risquées aux indépendants et à promouvoir massivement quelques rares - titres phares » explique Joëlle Farchy. Cela est une tendance lourde de l'économie des médias en général, du cinéma en particulier. Cependant, la période récente est le cadre d'un intérêt renouvelé des grands groupes pour ces créneaux. Ils y investissent par le biais de compagnies indépendantes acquises (Miramax, New Line, ParamountVantage) ou créées de toutes pièces (Fox Searchlight, Warner Independent Pictures). De même, l'investissement dans les cinématographies locales constitue une volonté de conquérir un créneau qui résiste et d'empocher des recettes qui autrement, restent en dehors de son atteinte. En effet, les résistances locales se concrétisent par des parts de marché maintenues dans un certain nombre de pays. Si les résultats de l'Inde, de la Corée ou de la France sont connus, des pays aussi disparates que l'Argentine (un quart des entrées pour le cinéma local en 2003), le Brésil (21 \% des entrées la même année), l'Allemagne (17\%) ou la Norvège (un peu moins de 19\%) maintiennent un noyau plus ou moins importants de spectateurs pour le cinéma local. Reprenant un argument ancien, Simona Benzakein, vice-présidente local production de Warner indique : « Puisque les films locaux marchent très bien, dans tous les pays, [les dirigeants de Warner] ont dit: «On est la major la plus indépendante, pourquoi on n'essaierait pas? » (Visco Comandini, $2006:$ I).

L'acceptation du concept de cinéma national par les Américains évoquée par Claude Miller doit donc être modulée. II n'empêche que le marché peine à assurer la promotion de la diversité rappelle Joëlle Farchy dès les premières lignes de son texte. Bien souvent, nonobstant avec une politique culturelle étatique, le marché est binaire, limité à la confrontation, Hollywood vs cinéma national. À ce sujet, le phénomène des productions localisées ne tend pas plus à « ouvrir le marché » qu'à conforter ce dualisme. L'exemple du débat français en 2003 autour du film Un long dimanche de fiançailles en est une illustration. Le film est financé par 2003 Productions, société créée en France par Warner Bros. Warner qui avait acquis les droits du livre de Sébastien Japrisot pour le tourner en anglais les rétrocède à Jean-Pierre Jeunet : «Pour nous, c'est une chance inouïe qu'un réalisateur de la trempe de Jean-Pierre Jeunet se soit intéressé à un roman que nous possédions. Que le film se fasse en France et en français témoigne de notre volonté d'aller jusqu'au bout de notre démarche de produire en Europe des films européens, c'est-à-dire en français, en espagnol et en italien », explique Francis Boespflug qui dirige Warner France ${ }^{10}$. Le film reçoit l'agrément du CNC, décision contestée

10 Écran total, 435 (09/10/02). 
par le Syndicat des Producteurs Indépendants (SPI) et l'Association des producteurs indépendants (API). Si le SPI compte parmi ses membres une partie des petites sociétés de production française, l'API est limitée à quatre compagnies: Gaumont, Pathé, UGC et MK2, soit les principales sociétés françaises. L'action menée par l'API traduit une volonté des « champions nationaux » de défendre leur pré carré. Nulle défense d'un cinéma national face à une menace étrangère ici, mais l'analyse que la production localisée initiée parWarner/2003 Productions consiste à capter pour une compagnie étrangères des recettes sur le territoire national réalisées par un film français. De fait, l'existence de politique de soutien en France ou en Europe, n'a guère eu pour effet de favoriser une diversité du spectacle cinématographique. Si le marché des États-Unis est un marché fermé aux films étrangers, les films des pays d'Europe centrale et orientale pour leur part ont pratiquement disparu des écrans de l'Union européenne : 0,054 \% dans les six années de 1996 à 200। ! Les films du pourtour méditerranéen s'octroient une place de 0,084 \% dans le même laps de temps et les films latino-américains «atteignent 》0,14\%. Comme le conclut l'étude de l'Observatoire européen de l'audiovisuel (OEA, 2002 : 4) : «C'est dire que pour les films des pays tiers autre que les États-Unis, la Canada, l'Australie et le Japon, le marché européen reste extrêmement fermé, plus fermé que le marché nord-américain ne l'est lui-même pour les films européens ».

\section{Conclusion}

Ce court vagabondage en cinéma autour de la glocalisation, des runaway et local productions illustre la complexité du phénomène de mondialisation en matière de cinéma. Loin d'être un processus univoque, c'est un processus fait d'à-coups, de chocs en retour, et de non-dits. Que les films soient tournés à Hollywood ou à Prague, qu'ils soient le fait de Warner, Gaumont ou Working Title, que des quotas soient érigés ou en déshérence, le mouvement brownien qui agite le cinéma mondial contemporain n'aboutit le plus souvent qu'à mettre en présence sur les marchés que deux cinématographies. La prise en compte de cette réalité doit conduire à réfléchir à la mise en place de politiques visant à conforter une véritable pluralité de l'offre et non le maintien d'un dualisme de l'offre.

\section{Références}

Appadurai A., 1990, « Disjuncture and difference in the global cultural economy », Public culture, 2(2), pp. I-24.

- 1996, Après le colonialisme, les conséquences culturelles de la globalisation, trad. de l'anglais par Fr. Bouillot, Paris, Payot, 2001. 
Chase K., 2000, « Globalization versus Localization : Cultural Protection and Trade Conflicts in the World Entertainment Industry », document préparatoire au panel Hollywood and Hegemony : Cinema, Capitalism and World Power, réunion annuelle de l'International Studies Association, Los Angeles, I 4- 18 mars.

Dacbert S., 1997, «Warner accompagne le prochain Claude Miller », Le Film français, 12 mai, p. 3

Dacbert S., Drouhaud S., 2004, « 2003 Productions persiste avec Ice3 et Josy Films », Le Film français, 9 janv., p. 5

Farchy J., Tardif J., 2006, Les enjeux de la mondialisation culturelle, Paris, Éd. Hors Commerce.

Frater P., 2007, « Bis'Eastern outposts », Variety, 12 févr.

Koszarski R., 1994, History of the American Cinema \#3. An Evening's Entertainment: the Age of the Silent Picture 1915-1928, Berkeley, University of California Press.

LaPorte N., 2004, « Local pix pique H'wood interest », Variety, I 3 sept.

Lev P., 1993, The Euro-American Cinema, Austin, University of Texas Press.

López Silvestre F., 2004, El paisaje virtual. El cine de Hollywood y el neobarroco digital, Madrid, Biblioteca Nueva.

Miller T., Govil N., McMurria J., Maxwell R., Wang T., 2005, Global Hollywood 2, Londres, British Film Institute.

Minguant, N, 2008, « Les stratégies d'exportation du cinéma hollywoodien (19662004) 》, thèse en études anglo-américaines, université Paris 10-Nanterre, en cours.

Mulard C., 1994, « Disney mise sur les films européens », Le Monde, I I oct.

Norden M., 1994, « Pathé Frères à l'époque du Trust » pp. 90- I 01 , in : Kermabon J., dir., Pathé premier empire du cinéma, Paris, Éd. du Centre Georges Pompidou.

O'Boyle M., 2007, « Brand New Thrust », Variety, 12 févr.

OEA (Observatoire européen de l'audiovisuel), 2002, « Étude pour la Conférence sur le secteur cinématographique et audiovisuel dans l'Union européenne et les pays tiers, Madrid, I8-19 avr.

Puttnam D., 1997, The Undeclared War (The Struggle for Control of the World's Film Industry), Londres, Harper et Collins.

Stolz J., 2004, « Eric Pleskow, quand Hollywood aimait l'Europe », Le Monde 19-20 déc.

Visco Comandini G., 2006, entretien avec Simone Benzakeim, non publié.

Warner J., Dickey C., 1996, «Together at Last », Newsweek, 13 mai.

Wright C., 2006, « Hollywood's Disappearing Act: International Trade Remedies to Bring Hollywood Home », Akron Law Review, vol.3 9, 3, p. 379, consultable sur http://papers.ssrn.com. 